\title{
Les mouvements de versant de la Montagne des Piniès (Drôme). Importance du rôle du contexte géologique
}

P. ANTOINE

P. DESVARREUX

A. GIRAUD

Lirigm

BP 53

38041 Grenoble cedex 9

\section{E. LEROI}

BRGM

117 av, de Luminy 13009 Marseille
Le graben de Mondorès au pied de la montagne des Piniès présente, sur une superficie très restreinte, un ensemble spectaculaire de mouvements de terrains de typologie très variée, Parmi ceux-ci, on remarque particulièrement I'affaissement d'une puissante falaise calcaire ( $50 \mathrm{~m}$ en une cinquantaine d'années) et deux importantes coulées boueuses, périodiquement réactivées dans la même période. L'origine de ces mouvements ainsi que les mécanismes de leur mise en place ne peuvent s'expliquer que par la reconstitution d'une histoire géologique particulièrement complexe appuyée par une auscultation sommaire. L'ensemble fournit un exemple remarquable du lien étroit qui existe entre les conditions géologiques d'un site et les mécanismes de la déformation gravitaire d'un versant, à une échelle et dans des conditions d'observation particulièrement favorables.

\section{The slope slides at Mont Piniès (Drôme-France). Importance of the geological context}

The Mondorès graben at the foot of Mount Piniès, displays, on a limited area, a spectacular set of landslides which typology is much varied. A huge sagging of a thick limestone cliff (amounting to about $50 \mathrm{~m}$ within half a century) and two important mud flows, periodically reactivated within the same span of time, are specially worthy of interest. The origin of these slope movements and their mechanisms of setting could only be understood through the reconstitution of a particularly complex geological history and with the help of a succinct monitoring. The whole offers a remarkable example of the close bonds which exist between the geological conditions of a site and the mechanisms of the gravitational deformation of a slope at particularly favourable scale and observation conditions.
} 


\section{Introduction}

Les mouvements du versant de la Montagne des Piniès, situé dans le département de la Drôme à $6 \mathrm{~km}$ à l'est de Boulc-en-Diois, présentent un certain nombre de particularités (Fig. 1). Tout d'abord, ils affectent à la fois des terrains de couverture et le substratum rocheux constitué de faciès très divers. Ensuite, ils se situent dans une zone remarquable sur le plan de la tectonique car il s'agit d'un fossé d'effondrement remanié lors de la surrection alpine. Enfin, les mouvements observés comportent un grand glissement de versant et des coulées boueuses. Seules ces dernières ont causé des inquiétudes entre 1978 et 1986 car elles menaçaient des habitations isolées. Le reste des mouvements concerne une zone inhabitée.

Un certain nombre d'études et de recherches ont été effectuées sur ce site entre 1986 et 1995 dont l'essentiel a été présenté dans les articles de Malatrait et. Sabatier (1996) et Girault (1997).

A. Malatrait et F. Sabatier ont reconstitué I'historique des mouvements, décrit ceux-ci et leur évolution. Ils ont présenté des hypothèses sur les mécanismes.

F. Girault a décrit les principaux résultats obtenus dans le cadre d'un contrat de plan État-région et d'un contrat avec la commission des Communautés européennes (HYCOSI). Il s'agit essentiellement de mesures topographiques par visées optiques et mesures GPS, de comparaison de divers modèles numériques de terrain entre 1972 et 1993, de mesures de déplacements de balises émettrices repérées par rapport à des satellites dont les positions sont connues avec exactitude. Mais les résultats de ces mesures n'ont pas été exploités en les intégrant au contexte géologique.

L'objet du présent article est d'établir la synthèse des données actuellement disponibles en géologie (nouvelles observations de terrain) et des principaux résultats de mesures effectuées dans le cadre du projet HYCOSI.

\section{2}

\section{Contexte géologique}

\section{1}

\section{Aperçu lithologique}

La série statigraphique locale est caractérisée par une alternance de formations marneuses ( $\propto$ Terres noires x de la base du Jurassique supérieur, marnes "jaunissantes " du Crétacé inférieur, marnes grisbleuté de l'Aptien, marnes rouges silto-argileuses de I'Oligocène) et calcaires (Tithonique, Barrêmo-Bédoulien). Ces dernières, dont l'épaisseur cumulée atteint 400 à $500 \mathrm{~m}$, se trouvent noyées au sein des marnes dont l'épaisseur totale est de l'ordre de 2500 à $3000 \mathrm{~m}$.

\section{2.}

\section{Évolution structurale}

L'histoire structurale du secteur considéré, polyphasée, s'échelonne du Trias au Tertiaire. La superposition des déformations résultantes, appliquées à un

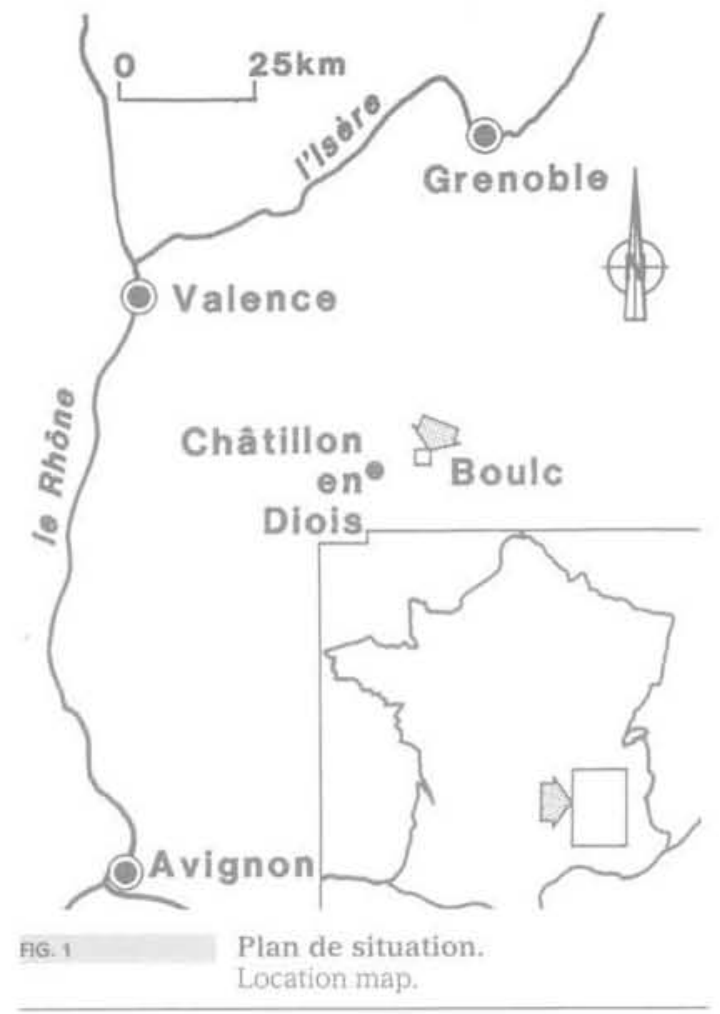

matériel hétérogène (alternance de niveaux rigides et déformables), a produit une structure particulièrement complexe dont dépendent largement les mouvements gravitaires récents.

A l'échelle régionale, le régime des déformations est essentiellement distensif entre le Trias supérieur et le Jurassique moyen. Cela entraine la formation de bassins subsidents séparés par des seuils.

Du Crétacé supérieur au Tertiaire prédomine, par contre, un régime compressif :

- avant le Sénonien se forment des plis d'axe sensiblement est-ouest témoignant d'un raccourcissement nord-sud:

- ces structures plissées sont accentuées à la limite Crétacé-Eocène et à l'Eocène inférieur :

- au Tertiaire (Oligocène et surtout Miocène), l'orogénie alpine proprement dite (phase de raccourcissement induisant un serrage sensiblement est-ouest) provoque le rejeu, en chevauchement ou en décrochement, d'accidents hérités des phases antérieures, lesquels sont fréquemment déformés. Seuls les accidents les plus récents (post-Miocène?) échappent, à ce type de déformation.

Dans le secteur étudié (Fig. 2), l'évolution structurale, ramenée à l'essentiel, se traduit par les faits suivants :

a) formation au Crétacé supérieur (avant le Sénonien), de l'anticlinal de Bonneval d'axe sensiblement est-ouest et tendance à l'extension dans cette direction :

b) accentuation de ce pli à la fin du Crétacé supérieur et au début du Tertiaire. L'aggravation résultante de l'étirement axial est à l'origine de l'individualisation (à l'échelle régionale) du graben de Mondorès de direction $\mathrm{N} 20^{\circ}$ environ; une émersion générale de la zone se produit alors :

c) à l'Oligocène, la dépression ainsi formée permet le dépôt de la molasse rouge continentale associant des 
niveaux conglomératiques polygéniques et silto-argileux (ces derniers nettement plus abondants). On notera que, localement, la présence de la molasse rouge est une singularité spécifique au graben de Mondorès ;

d) le serrage alpin post-Oligocène entraine la déformation des accidents bordiers du graben (Fig. 2 et 3 ). Ceux de la limite ouest sont alors redressés quasiment à la verticale tandis que ceux de la limite est sont déversés vers l'ouest (chevauchements). Cette différence d'attitude est bien visible sur la coupe de la figure 6. Elle peut s'expliquer par un gradient décroissant de la sollicitation, d'est en ouest, accompagné de bourrage et de clivages dans les séries marneuses largement prédominantes au sein du graben ;

e) les phases ultimes de serrage alpin sont responsables d'un coulissage longitudinal le long du graben de Mondorès (décrochement dextre - Fig. 2) et de l'apparition de failles verticales à rejet quasiment nul (fractures sommitales de la Montagne des Piniès délimitant le tassement affectant cette dernière),

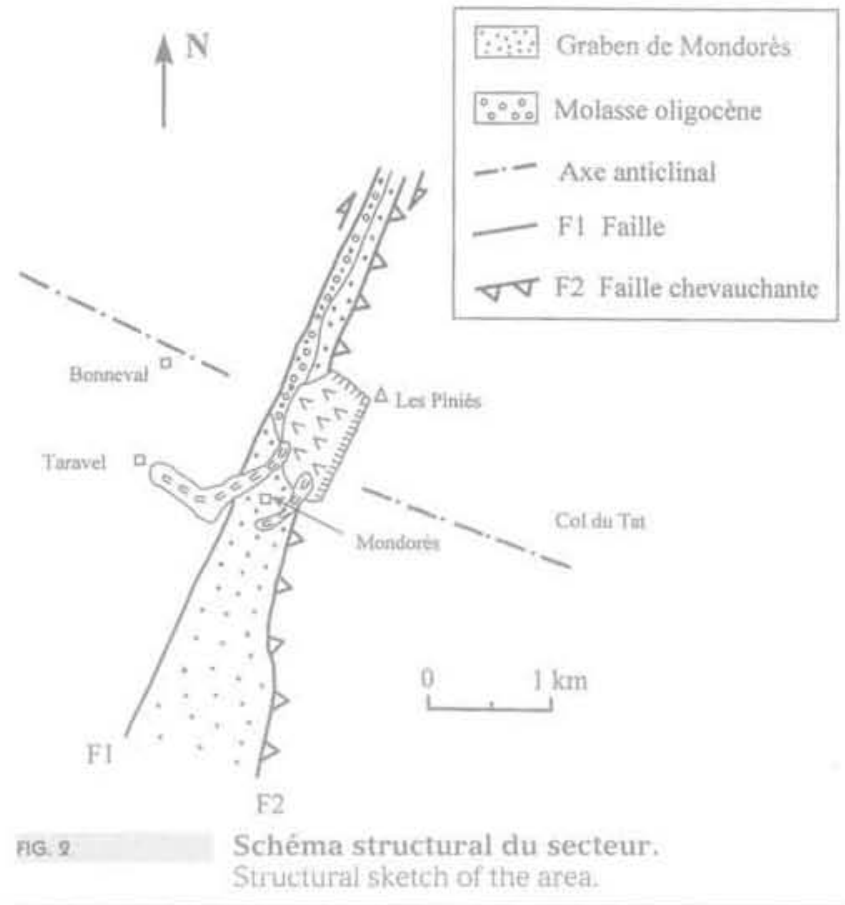

\section{Description et historique des mouvement (Fig. 3)}

Sur l'ensemble du graben de Mondorès, $80 \%$ de la superficie sont affectés de mouvements de versants. Cette densité exceptionnelle est à l'évidence une conséquence des conditions lithologiques et structurales. Ceci se double en outre d'une diversité typologique résultant de mécanismes très différents puisque l'on observe, étroitement associés : des coulées, un tassement et des glissements auxquels se surimposent des écoulements localisés et des chutes de blocs.

\section{Les coulées}

Celles-ci confèrent au site son aspect spectaculaire par leur volume et leur différence de teinte. La coulée de Taravel (la plus à l'ouest) est alimentée surtout par la molasse rouge oligocène. La coulée de Mondorès (la plus à l'est) est alimentée par les ensembles marneux sombres des a Terres noires $)$ et de l'Aptien.

\section{2}

\section{Le tassement des Piniès}

Un autre trait tout à fait caractéristique du site est l'existence de falaises hautes d'une cinquantaine de mètres formant un dièdre largement ouvert à l'ouest, lequel limite, dans cette direction, le sommet des Piniès. Celui-ci s'est trouvé dégagé récemment par l'affaissement d'un volumineux coin rocheux (de l'ordre de 5 millions de $\mathrm{m}^{3}$ ), le tassement des Piniès, constitué de calcaires de la base du Berriasien, des calcaires du Tithonique et du Kimmeridgien supérieur et des marno-calcaires et marnes du Kimmeridgien inférieur. Les observations détaillées de terrain ne montrent pas que des matériaux situés plus bas dans la série aient participé à ce mouvement.

\section{3}

\section{Les glissements}

L'espace subsistant entre les deux types de mouvements précédents est affecté de glissements formés au détriment du substratum marneux. Ceux-ci sont la conséquence des modifications morphologiques engendrées par le départ des coulées et l'accentuation du tassement des Piniès. On verra plus loin que ces glissements peuvent être rattachés à la partie inférieure du mouvement d'ensemble.

\section{4}

\section{Aperçu chronologique}

Il convient de souligner que l'essentiel des mouvements ci-dessus s'est développé ou plutôt réactivé depuis seulement une cinquantaine d'années comme en attestent les photographies aériennes et les témoignages locaux.

Des coulées sont signalées vers 1930 et les photos de 1948 (les plus anciennes dont nous disposons) montrent bien l'existence de coulées dans les talwegs de Taravel et de Mondorès. Par contre, le tassement des Piniès est au stade initial d'une simple crevasse sommitale sans qu'on en connaisse la date d'apparition. Néanmoins, l'examen attentif de ces photos montre que des phénomènes de glissements affectant le substratum rocheux se sont déjà produits dans le passé dans le versant ouest de la Montagne des Piniès entre la cote $1400 \mathrm{~m}$ et la cote $1100 \mathrm{~m}$, mais leurs formes sont passablement estompées.

Entre 1948 et 1977, l'observation des photographies aériennes (1956, 1971, 1977) révèle l'absence d'activité des coulées, attestée par leur végétalisation progressive. On note par contre une individualisation plus marquée du tassement des Piniès. 


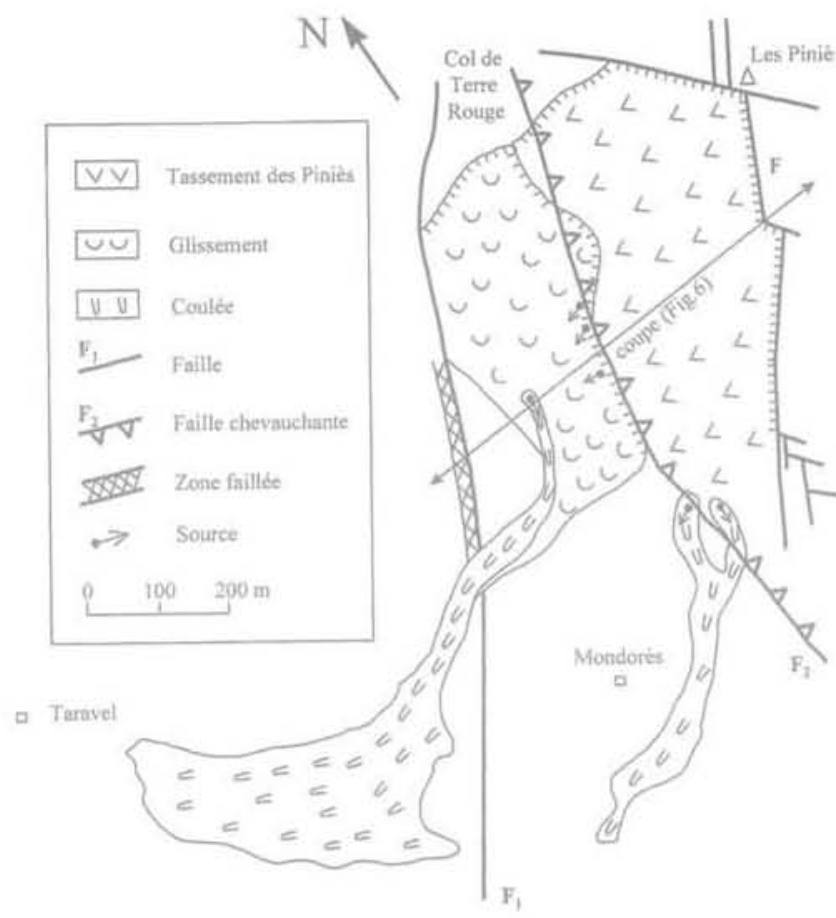

FiG. 3

Carte schématique du site.

Schematic map of the site.

L'hiver 1977-1978 est marqué par l'accélération brutale de ce dernier, lequel s'abaisse de plusieurs dizaines de mètres (témoignages locaux). Cette évolution est suivie très rapidement (mars-avril 1978) d'une réactivation spectaculaire de la coulée de Taravel laquelle se rapproche dangereusement de la ferme du même nom. On peut donc noter une certaine concomitance entre l'accentuation du tassement et la réactivation des coulées. Ce processus se poursuit probablement à l'heure actuelle comme en témoigne la désorganisation progressive du tassement des Piniès et l'occurrence de crises en 1985, 1986 et 1991 dans l'activité des coulées. Celles-ci se produisent généralement au printemps.

\section{Hydrogéologie}

L'eau souterraine est présente en relative abondance dans le graben de Mondorès comme en témoignent le volume des coulées boueuses de Taravel et de Mondorès ainsi que l'existence de nombreuses sources pérennes (Fig. 3 et 4).

Cependant, le bassin versant correspondant strictement au graben est extrêmement réduit (de l'ordre de $0,5 \mathrm{~km}^{2}$ ), et son substratum est essentiellement marneux, donc a priori peu perméable.

En toute hypothèse, les quantités d'eau infiltrée directement depuis la surface du graben sont certainement peu importantes. On est donc obligé d'envisager une alimentation plus lointaine à partir d'un bassin versant plus étendu. En dehors de la molasse rouge, dont la perméabilité reste néanmoins faible, les seuls aquifères notables sont représentés par les formations calcaires, notamment par le Tithonique et le BarrêmoBédoulien. De fait, on a observé, sur les limites latérales du tassement et sur certains plans de fractures, qu'on recoupe fréquemment des chenaux de type karstique.
On peut donc admettre que les transferts hydrauliques sont possibles entre le réservoir calcaire des Piniès et le graben de Modorès via les limites mèmes du tassement.

T. Bogaard (1996) par d'autres types d'investigations (analyses chimiques des eaux, exploitation des pièzomètres courts) confirme cette interprétation.

En observant (Fig. 4) la position des sorties d'eau principales on peut faire deux remarques :

- un grand nombre d'entre elles se situent sur une ligne de direction $\mathrm{N} 120^{\circ}-130^{\circ}$ s'élevant du sud-est au nord-ouest de $1110 \mathrm{~m}$ (Mondorès) à $1200 \mathrm{~m}$ (sous le Col de Terre rouge). Cette ligne peut représenter la sortie du mouvement principal :

- la localisation de la zone de départ des deux coulées de Taravel et Mondorès se situe pratiquement sur la ligne précédente, ce qui tend à montrer que l'eau de formation des coulées transite par ce mouvement principal (Fig. 4).

Enfin, les mesures de pression interstitielle effectuées dans le sondage SD, par des cellules à cordes vibrantes ont donné les résultats qui ont été résumés dans le tableau I. On peut prèciser que la coulée, au niveau du sondage SC, voisin, a une épaisseur de $5,5 \mathrm{~m}$, repérée par le cisaillement du tube inclinométrique.

La coupe de ce sondage montre qu'on rencontre les marnes bleues, rouges et vertes tectonisées de $6,5 \mathrm{~m}$ à $25 \mathrm{~m}$. Les trois cellules sont donc implantées dans le substratum marneux du fossé de Mondorès. Elles sont soigneusement isolées par des bouchons d'argile expansée. Les deux séries de mesures des 22 et 27 novembre 1994 montrent que toutes ces pressions sont indicatives d'un seul et même écoulement dont la pente est de l'ordre de $13^{\circ}$, c'est-à-dire exactement celle du terrain naturel. On peut constater deux points qui à notre avis sont très importants :

- le remplissage marneux du graben, tout au moins au niveau du sondage SD, est très fracturé et saturé sur au moins $25 \mathrm{~m}$ d'épaisseur :

- les quelques mesures de niveau d'eau effectuées montrent qu'a l'automne 1994 ce dernier est situé entre 2.5 et $3 \mathrm{~m}$ de profondeur. Des remontées plus importantes ne sont pas exclues car l'automne 1994 n'a pas connu des précipitations exceptionnelles. Par conséquent, l'activité des coulées peut être liée aussi bien aux quantités d'eau ressortant du glissement principal (sans influence particulière de I'hydrogéologie des talwegs) qu'à des remontées du niveau d'eau suffisamment près de la surface sous le fond des talwegs. Dans ce second mécanisme, on peut aboutir à une mobilisation de tout le fond du talweg sur 4 à $5 \mathrm{~m}$ d'épaisseur, sans qu'il y ait forcément d'activité importante dans le mouvement d'ensemble.

TABLEAU1 Valeurs de pression d'eau (en m) audessus des cellules du sondage SD1. Water pressure values above the cells placed in borehole SD1.

\begin{tabular}{l|r|r|r} 
Cellule $^{\circ}$ & 10021 & 10022 & \multicolumn{1}{c}{9986} \\
\hline Profondeur/TN & $-6,7 \mathrm{~m}$ & $-11,0 \mathrm{~m}$ & $-23,6 \mathrm{~m}$ \\
\hline $22 / 11 / 1994$ & $3,61 \mathrm{~m}$ & $7,75 \mathrm{~m}$ & $19,73 \mathrm{~m}$ \\
\hline $27 / 11 / 1994$ & $3,56 \mathrm{~m}$ & $7,64 \mathrm{~m}$ & $19,62 \mathrm{~m}$ \\
\hline $21 / 02 / 1995$ & & $8,06 \mathrm{~m}$ & \\
\hline
\end{tabular}




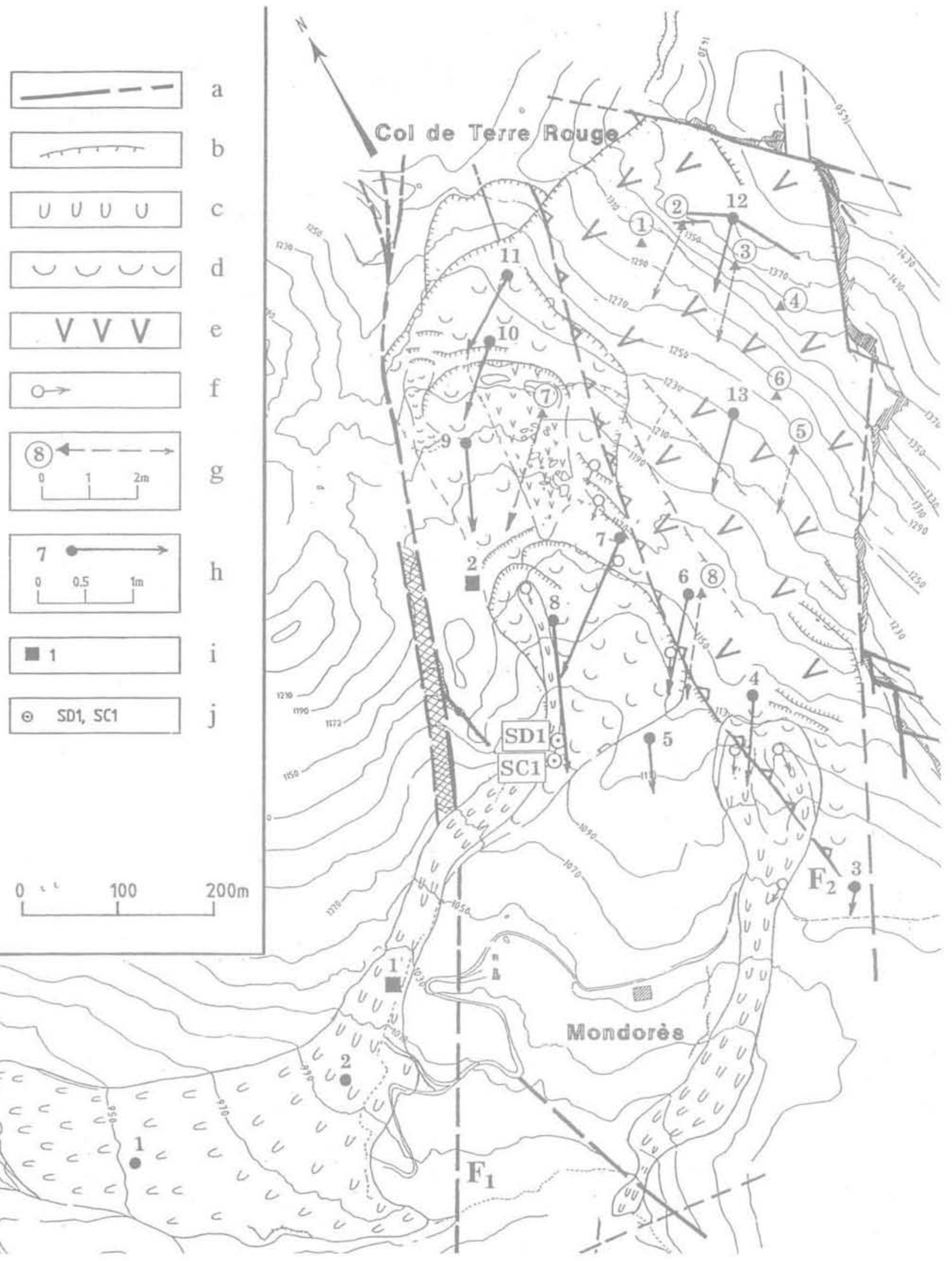

FIG.4 Implantation de l'auscultation et des reconnaissances.

a. Faille, b. niche d'arrachement, c.- coulée, d. glissement, e. tassement, f. source, g. cible IGN et son déplacement (28-06-93 à 10-08-95), h. point GPS et son déplacement (06-93 à 04-94), i. balise DORIS, j. sondage.

Surveving and monitoring network.

a. Fault, b. Main scarp, c. Mud flow, d. slide, e. sagging, f. spring, g. IGN target and its moving (28-06-93 to 10-08-95), h. GPS point and its moving (06-93 to 04-94), i. DORIS beacon, j. Borehole. 


\section{Apport des mesures de déplacements}

Parmi les diverses mesures effectuées dans le cadre du projet HYCOSI, nous retiendrons :

- les mesures topographiques classiques en X. Y, Z sur 7 cibles IGN entre le 20/05/1992 et le 10/08/1995 (5 mesures). La cible 6 a ẻté installée mais jamais mesurée :

- les mesures GPS en X, Y, Z sur 13 points GPS entre juin 1993 et avril 1994 (4 mesures) ;

- les mesures IGN en X, Y, Z sur deux balises DORIS les 20/05/1992 et le 12/08/1992.

\section{1}

\section{Directions de vecteurs de déplacements}

Sur la figure 4 on a reporté les directions et modules des déplacements horizontaux. On s'est heurtè à la difficulté de la non-concordance des dates de mesures sur les cibles IGN et les points GPS. On a choisi les périodes suivantes :

- 28/06/1993 au 10/08/1995 pour les cibles IGN,

- juin 1993 à avril 1994 pour les points GPS.

On peut constater sur la figure 5 que les vitesses moyennes des divers témoins durant ces deux périodes sont du même ordre de grandeur. Comme la période d'observation sur les cibles IGN est 2,5 fois celle des points GPS, en utilisant une échelle double pour les déplacements des points GPS par rapport aux cibles IGN, on obtient des données un peu plus homogènes qui montrent deux éléments intéressants :

- pour les points placés à l'amont de la ligne des sources, les divers vecteurs de déplacements sont sensiblement parallèles entre eux et de direction $\mathrm{N} 40^{\circ}$, donc pratiquement perpendiculaires à la direction de la famille de fractures N $130-140^{\circ}$ dont l'une d'entre elles contrôle le tassement sommital. Ceci montre qu'il s'agit d'un mouvement profond, commandé par des discontinuités du massif rocheux;

- alors qu'en amont de la ligne de sources les vecteurs de déplacements pouvaient n'avoir aucun lien avec la direction des lignes de plus grande pente, en aval il n'en va pas de même. Les tếmoins GPS 8 et 9 ont des vecteurs de déplacements beaucoup plus proches des lignes de pente, montrant que dans la bande large de $100 \mathrm{~m}$ environ, coincée entre la bordure occidentale du graben de Mondorès et l'extrémité du mouvement principal les déplacements se font dans une direction N $20^{\circ}$.

\section{2}

\section{Modules des vecteurs de déplacements}

Sur la figure 5 on a représenté les valeurs des modules de divers vecteurs de déplacements $\sqrt{\Delta \mathrm{x}^{2}+\Delta \mathrm{y}^{2}+\Delta \mathrm{z}^{2}}$ toutes directions confondues et on peut faire d'autres remarques intéressantes :

- si on considère les déplacements de points situés sur les mèmes plans longitudinaux (c'est-à-dire les mèmes plans verticaux contenant les déplacements) on constate quíls sont semblables, par exemple GPS 6 et 13, GPS 12 et 7, ce qui confirme l'unicité du mouvement;

- si on considère les déplacements de points situés sur une même horizontale, on constate que les vitesses maximales dans le mouvement principal se trouvent au centre (témoins GPS 7 et 12). Plus on va vers l'est ou l'ouest, plus les vitesses diminuent;

- entre juin 1993 et mars 1994 il n'y a pas eu de coulées puisque les térnoins GPS 1 et 2 n'ont pas bougé. Or, pendant ce temps, les mouvements ont été actifs en partie supérieure $(0,70$ à $1,36 \mathrm{~m}$ de déplacements). Le point GPS 8 ayant eu néanmoins une activité durant cette période $(1,4 \mathrm{~m})$ on peut conclure que les coulées s'arrêtent plus ou moins haut dans le talweg en fonction des saisons :

- les mouvements ne se font pas à vitesse constante dans le temps mais le petit nombre de mesures ne permet pas de déterminer avec précision les fluctuations de vitesse. Tout au plus, on peut constater que les vitesses moyennes entre le 12/08/1992 et 28/06/1993 varient de 24 à $36 \mathrm{~cm} / \mathrm{mois}$ alors qu'elles sont de l'ordre de $9 \mathrm{~cm} / \mathrm{mois}$ entre le 28/06/1993 et le 10/08/1995; - les deux balises DORIS (voir leur implantation à la figure 4) ont été mesurées par lTGN entre le 20/05 et le 12/08/1992. La balise 2, située à $1160 \mathrm{~m}$ d'altitude, dans la bande de direction $\mathrm{N} 20^{\circ}$ située sous le Col des Terres Rouges, s'est déplacée de $1,06 \mathrm{~m}$, donc plus rapidement que les cibles IGN situées sur le mouvement principal (Fig. 5). Durant la mème période, la balise 1 s'est déplacée de près de $47 \mathrm{~m}$, ce qui montre qu'il y a eu une activité de coulées indubitable. Les mesures de détail DORIS fournies dans le compterendu HYCOS sont difficile à interpréter. Néanmoins, il semble que l'essentiel de l'activité des coulées se soit concentré entre le 16 et le 28/06/1992, à un moment de précipitations importantes (257 $\mathrm{mm}$ en juin 1992 à Chatillon-en-Diois - Fig. 5). On a donc ici un indice objectif de la concomitance des coulées avec les précipitations abondantes et une certaine activité du mouvement principal.

\section{Inclinaison des vecteurs de déplacements}

On a reporté sur la coupe géologique synthétique de la figure 6 les vecteurs de déplacements obtenus sur les cibles IGN ou les points GPS durant les deux périodes déjà précisées. On donne d'autre part au tableau II les résultats des mesures planimétriques et altimétriques obtenus sur tous les points, permettant de mesurer l'inclinaison des vecteurs de déplacement $\alpha$ avec thorizontale.

On remarque que les inclinaisons des vecteurs de déplacement sont fortes pour les points les plus élevés (IGN 2, 3, 4, GPS 12) et qu'elles décroissent ensuite pour devenir horizontale et même légèrement remontantes (IGN 7 et 8, GPS 4, 6, 7, 8). Le passage entre ces inclinaisons extrêmes semble se faire assez rapidement puisqu'aux points IGN 5 et GPS 13 I'inclinaison est encore voisine de $45^{\circ}$.

On peut remarquer également que les inclinaisons des déplacements de IGN 1 et GPS 11 sont voisines de $30^{\circ}$, ce qui n'est pas conforme au comportement des points précédents. Enfin, on note que si les déplace- 


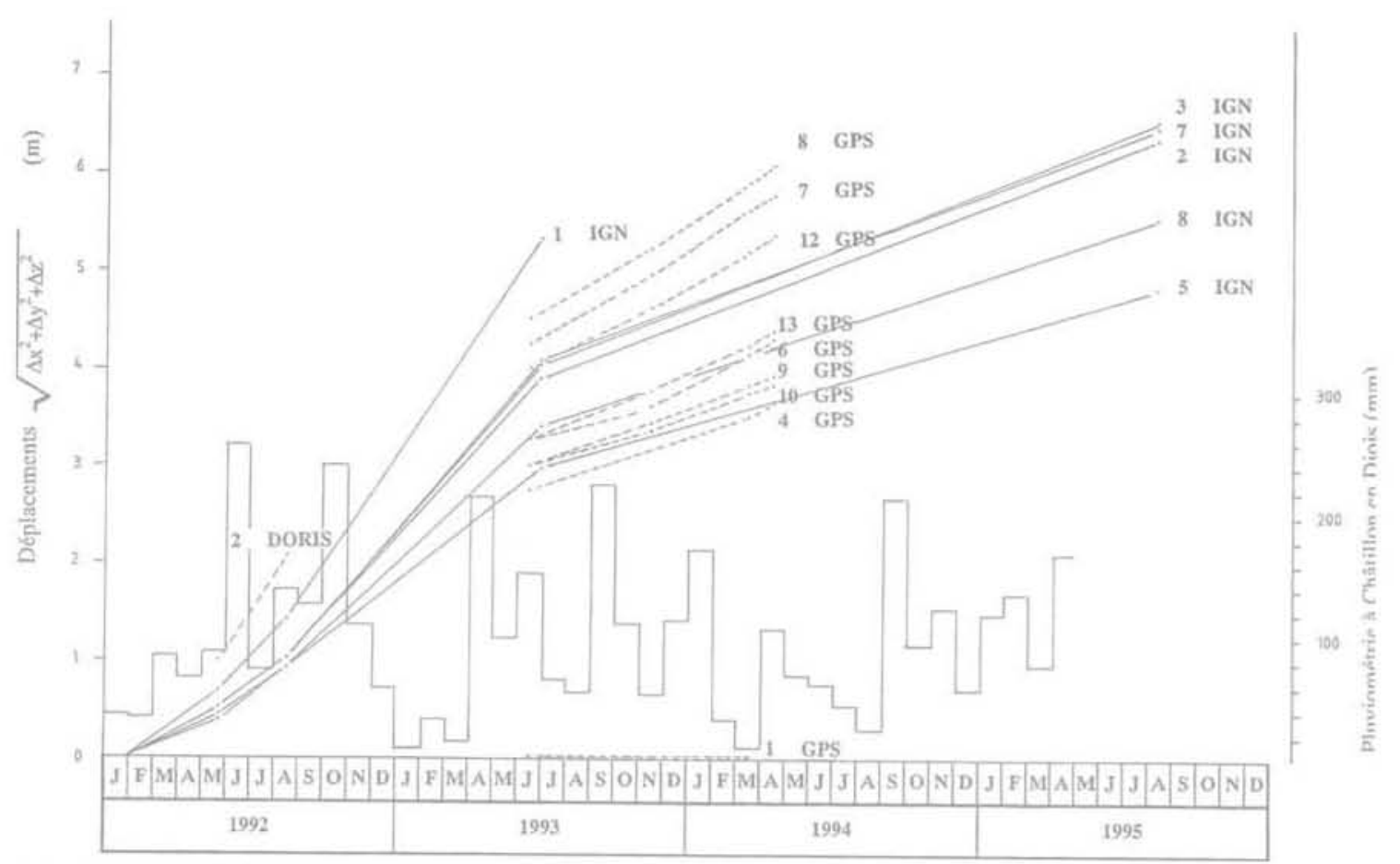

HG.5 Déplacements cumulés et pluviométrie.

Cumulated shifting and rainfall.

TABLEAUII Déplacements planimétriques et altimétriques.

Planimetric and altrimetric shift.

\begin{tabular}{l|l|c|c|c} 
Témoins & \multicolumn{1}{|c|}{ Période } & $\begin{array}{c}\sqrt{\Delta x^{2}+\Delta y^{2}} \\
(m)\end{array}$ & $\Delta z$ & $\alpha$ \\
\hline IGN 1) & $27.01 .92 / 28,06.93$ & $(4,53)$ & $(-2,77)$ & $-31^{\circ}$ \\
\hline IGN 2 & $28.06 .93 / 10.08 .95$ & 1,65 & $-1,84$ & $-48^{\circ}$ \\
\hline IGN 3 & $28.06 .93 / 10.08 .95$ & 1,54 & $-2,00$ & $-52^{\circ}$ \\
\hline IGN 4) & $27.01 .92 / 28.06 .93$ & $(2,32)$ & $(-3,54)$ & $-57^{\circ}$ \\
\hline IGN 5 & $28.06 .93 / 10.08 .95$ & 1,29 & $-1,34$ & $-46^{\circ}$ \\
\hline IGN 7 & $28.06 .93 / 10.08 .95$ & 2,37 & $-0,33$ & $-8^{\circ}$ \\
\hline IGN 8 & $28.06 .93 / 10.08 .95$ & 2,12 & $-0,06$ & $2^{\circ}$ \\
\hline GPS 3 & $06.93 / 04.94$ & 0,24 & $-0,057$ & $-13^{\circ}$ \\
\hline GPS 4 & $06.93 / 04.94$ & 0,87 & $-0,024$ & $-2^{\circ}$ \\
\hline GPS 5 & $06.93 / 04.94$ & 0,56 & $-0,44$ & $-38^{\circ}$ \\
\hline GPS 6 & $06.93 / 04.94$ & 1,01 & $+0,017$ & $+1^{\circ}$ \\
\hline GPS 7 & $06.93 / 04.94$ & 1,50 & $+0,092$ & $+4^{\circ}$ \\
\hline GPS 8 & $06.93 / 04.94$ & 1,56 & $+0,085$ & $+3^{\circ}$ \\
\hline GPS 9 & $06.93 / 04.94$ & 0,87 & $-0,322$ & $-20^{\circ}$ \\
\hline GPS 10 & $06.93 / 04.94$ & 0,82 & $-0,126$ & $-9^{\circ}$ \\
\hline GPS 11 & $06.93 / 04.94$ & 0,97 & $-0,544$ & $-29^{\circ}$ \\
\hline GPS 12 & $06.93 / 04.94$ & 0,80 & $-1,08$ & $-53^{\circ}$ \\
\hline GPS 13 & $06.93 / 04.94$ & 0,81 & $-0,774$ & $-44^{\circ}$ \\
\hline & & & & \\
\hline
\end{tabular}

ments de GPS 9 se font sensiblement parallèlement au terrain naturel, il n'en va pas de même pour le point GPS 8, qui remonte, alors que la pente du terrain à son voisinage est de l'ordre de $20^{\circ}$. Cette contradiction avec les autres données sur cette zone n'a pas trouvé pour l'instant d'explication.

Sur la figure 6 on a tracé une hypothèse de surface de mouvement principal prenant en compte les éléments ci-dessus, Le brusque changement de pente de la surface de glissement à la verticale du témoin GPS 13 pourrait ètre en liaison avec l'existence, en profondeur, d'une lame de calcaires (flanc inverse du synclinal déversé de Mondorès constitué de Barrémo-Bedoulien).

L'essentiel des matériaux participant au mouvement d'ensemble serait constitué de Jurassique supérieur (Terres Noires - Sequanien - Kimmeridgien et Tithonique). La base des mouvements d'ensemble fournirait en eau et en matériaux les deux zones de départ de coulées de Taravel et Mondorès. La butte de Mondorès. large de moins de $100 \mathrm{~m}$ à ce niveau, constitue une butée relativement peu efficace, les terrains la constituant étant repoussés par le mouvement d'ensemble (point GPS 5).

\section{6}

\section{Synthèse}

Les quelques remarques ci-dessus permettent alors de fournir les précisions suivantes sur l'ensemble des mouvements de la montagne des Piniès.

La réactivation à laquelle on assiste depuis 1948 ne constitue pas le début d'un mouvement car les observations de terrain et sur photos aériennes montrent 

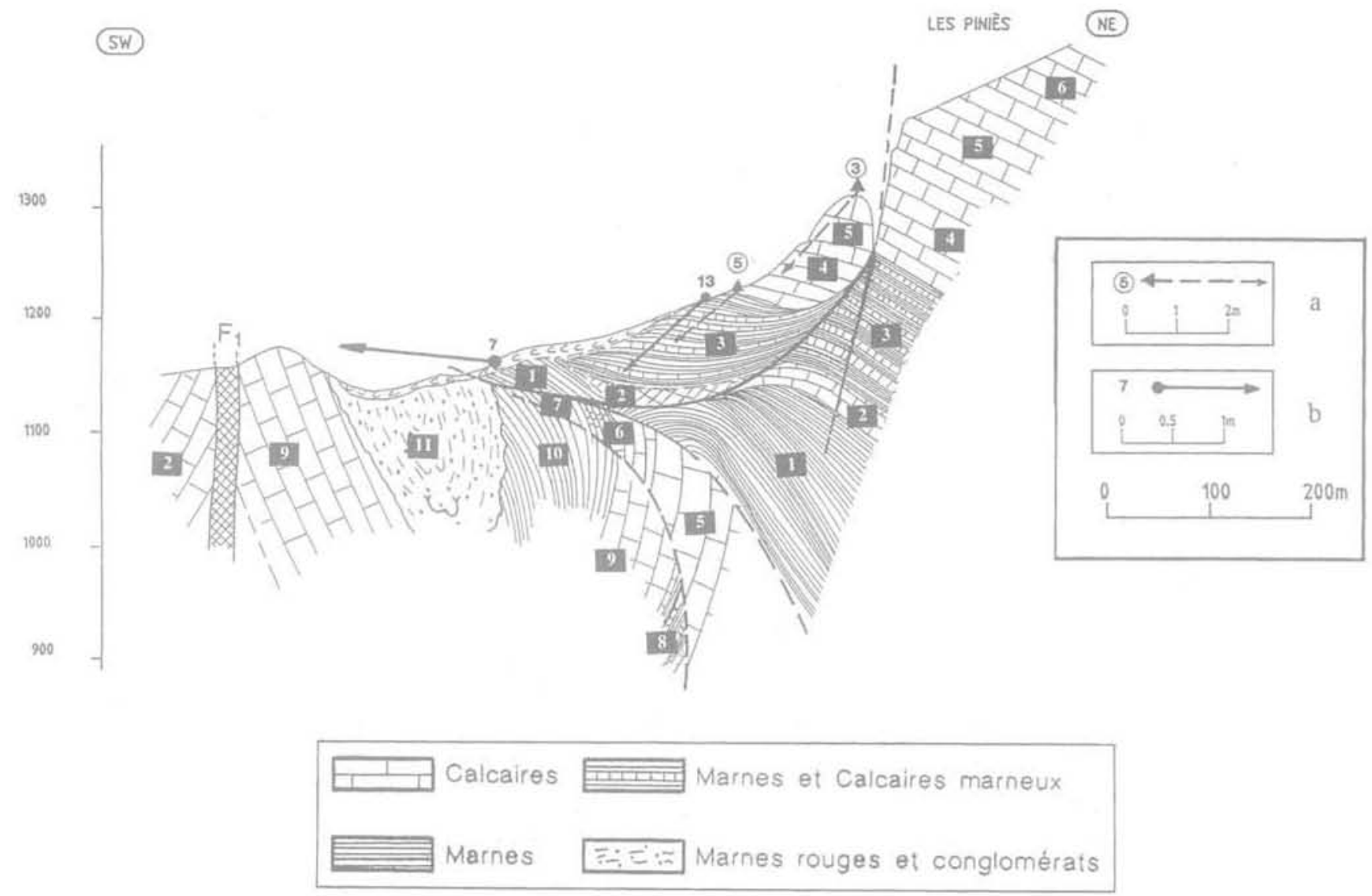

FG.6. Coupe géologique interprétative.

a. cible IGN et son déplacement (28-06-93 à 10-08-95), b. point GPS et son déplacement (06-93 à 04-94).

1. Callovo-oxfordien, 2. Séquanien, 3. Kimmeridgien inférieur. 4. Kimmeridgien supérieur, 5 . Títhonique.

6. Berriasien, 7. Valanginien, 8. Hauterivien. 9. Barrémo-Bedoulien, 10. Albo-Aptien, 11. Oligocène.

interpreted geotechnical section.

a. IGN target and its moving (28-06-93 to 10-08-95), b. GPS point and its moving (06-93 to 04-94). 1. Callovo-Oxfordian,

2. Sequanian, 3. Lower Kimmeridgian. 4. Upper Kimmeridgian, 5. Tithonian, 6. Berrasian. 7. Valanginian,

8. Hauterivian, 9. Barremo-Bedoulian, 10. Albo-Aptian, 11. Oligocene.

clairement qu'il y avait eu avant cette date d'autres phénomènes d'instabilité.

Il existe une relation entre le mouvement d'ensemble et les coulées. Ces dernières prennent naissance précisément à la base du premier et l'eau qui constitue les coulées provient vraisemblablement, en grande partie, du mouvement principal. L'alimentation de ce dernier intéresse un bassin versant plus grand que le simple bassin topographique du vallon de Terre Rouge, probablement par des cheminements karstiques dans les formations calcaires du Jurassique supérieur.

Cependant, les deux mécanismes ne sont pas exactement concomitants puisqu'on a mesuré des déplacements non négligeables (de l'ordre de 1 à $2 \mathrm{~m}$ ) du mouvement d'ensemble, sans coulées. A l'inverse, on ne peut exclure des coulées se produisant sans mouvement d'ensemble par remontée du niveau d'eau près de la surface. Ce niveau d'eau a été mesuré 2 à 3 fois seulement à une profondeur de 2,5 à $3 \mathrm{~m}$, et il affecte le remplissage argileux fissuré du talweg sur $25 \mathrm{~m}$ d'épaisseur.

Le mouvement d'ensemble doit intéresser une épaisseur de 50 à $100 \mathrm{~m}$ de terrains. Ses limites sont déterminées par des réseaux de discontinuités du Massif des Piniès, en particulier des plans de fractures de directions $\mathrm{N} 10-15^{\circ}$ et $\mathrm{N} 130^{\circ}$. Sa base doit être consti- tuée par une écaille rocheuse résistante, probablement des calcaires du Barrêmo-Bedoulien situés au moins à $50 \mathrm{~m}$ de profondeur. Le rôle de cette écaille (dont l'existence n'est qu'une hypothèse probable) est très important car elle limite le mouvement d'ensemble dans son extension en profondeur. En effet, l'essentiel du remplissage du graben à cet endroit est marneux. C'est l'abondance des marnes qui facilite le mouvement d'ensemble, mais c'est cette lame qui le maintient dans les limites acceptables (Fig. 6).

\section{7}

\section{Conclusion}

L'étude des mouvements de terrains du versant ouest de la Montagne des Piniès illustre remarquablement les rapports étroits qui existent entre le contexte géologique du site et les conditions géotechniques de stabilité des versants. Il apparaît clairement que l'auscultation seule (Girault F., 1997) ne peut fournir qu'un élément de description des phénomènes, au même titre que l'étude géologique de surface (Malatrait A., Sabatier F., 1996). Il est absolument nécessaire de combiner les deux approches pour espérer comprendre la nature et l'origine de la déformation des versants. L'intérêt du site de Mondorès-Les Piniès réside dans le fait qu'une 
histoire des déformations géologiques particulièrement riche en évènements conduit à un modèle de versant très complexe. L'établissement de ce dernier ne peut résulter de la seule approche géologique classique (cartographie très détaillée) car le dessin de la coupe interprétative doit faire la part de trop nombreuses hypothèses et laisse certaines questions sans réponse. La présence d'un vestige de flanc inverse du synclinal de Mondorès, rigide, car formé principalement de calcaires, fournit un bon exemple. L'analyse de la déformation, par le biais de l'auscultation pourtant sommaire qui a été réalisée, conforte cependant ce qui jusque-là n'est qu'une hypothèse géologique parmi d'autres. Il est en effet nécessaire qu'un niveau résistant existe à faible profondeur pour expliquer les variations de plongement des vecteurs déplacement telles qu'elles ont été mesurées. Ceci montre bien que, dans ce domaine particulier de la géotechnique (comme dans tous les autres), il ne faut jamais perdre de vue que la bonne compréhension de la structure et de l'histoire géologique d'un site est un préalable indispensable, ce qui apparait trop souvent ignoré.

\section{$\overline{\text { Bibliographie }}$}

Bogaard T. Van Asch T. - $\alpha$ Geophysical and hydrochemical investigation of a complex large-scale landslide in southern France 1. Proc. 7 th International Symposium on Landslides, Trondheim. Norway, vol. 2, 1996, p. 643-647,
Girault F. - « Auscultation du glissement du versant ouest de la montagne des Piniès dans la Drôme (France) n. Revue Francaise de Géotechnique, n* 79, 1997, p. 13-18

Malatrait A. Sabatier F. - " Le glissement de la montagne des Piniès à l'origine des coulées de Boulc-en-Diois (Drôme) Evolution et mécanismes n. Revue Fran. caise de Géotechnique, n*74, 1996, p. 4553. 